\title{
Weiterbildungskurse in Ungarn
}

Die Kurse im Csipkerozsa-Parkhotel in Süd-Ungarn erfreuen sich einer grossen Beliebtheit, zunehmend auch bei Kandidaten aus anderen Ländern. Die Supervisions- und Intensiv-Trainingskurse mit nur maximal 4-6 Teilnehmern füllen eine Marktlücke. Jeder Teilnehmer arbeitet dabei an einem eigenen modernen Gerät. In den 3-tägigen Kurzkursen können so $30-40$, in den Wochenkursen rund 80-90 Untersuchungen an Probanden und Patienten ermöglicht werden. Auch für Teilnehmer, welche am Abdomen und an den Weichteilen üben wollen, können entsprechende Patienten gestellt werden.

Die Grund- und Aufbaukurse erfüllen die Normen und sind SGUM-anerkannt. Alle anderen Kurse können auch als Refresherkurse angerechnet werden.

Neu sind im Jahr 2017 sogenannte „Chränzli-Kurse“, welche speziell zur Vertiefung von Themen gedacht sind und als
Zielpublikum bereits erfahrenere Sonografen ansprechen wollen.

Neben der Theorie und Praxis im Gebiet des Ultraschalls kommt aber auch die Freizeit nicht zu kurz und ein umfangreiches Angebot steht im Parkhotel zur Verfügung.

Gesucht werden auch Kursleiter, welche hier eigene Kurse (auch in anderen Fachgebieten) durchführen möchten. Die gesamte Logistik und Infrastruktur sowie Patienten können zur Verfügung gestellt werden. Auskunft erteilt gerne beat. dubs@sono-glattpark.ch.

Die Liste der Kurse wurde bereits im Heft 4-2016 abgebildet.

Weitere Kurse, insbesondere auch ein Interventionskurs sind noch geplant, Einzelheiten folgen auf der Webseite.

Das gesamte Angebot und die Einzelheiten zu den Kursen sind unter www. ungarnkurse.ch im Internet aufgeschaltet.

Beat Dubs 